\title{
Studies of a Next-Generation Silicon-Photomultiplier-Based Time-of-Flight PET/CT System
}

\author{
David F.C. Hsu ${ }^{1,2}$, Ezgi Ilan ${ }^{3,4}$, William T. Peterson ${ }^{5}$, Jorge Uribe ${ }^{5}$, Mark Lubberink ${ }^{3,4}$, and Craig S. Levin ${ }^{1,2,6,7}$ \\ ${ }^{1}$ Electrical Engineering, Stanford University, Stanford, California; ${ }^{2}$ Radiology, Stanford University, Stanford, California; ${ }^{3}$ Nuclear \\ Medicine and PET, Department of Surgical Sciences, Uppsala University, Uppsala, Sweden; ${ }^{4}$ Medical Physics, Uppsala University \\ Hospital, Uppsala, Sweden; ${ }^{5}$ GE Healthcare, Waukesha Wisconsin; ${ }^{6}$ Physics, Stanford University, Stanford, California; and \\ ${ }^{7}$ Bioengineering, Stanford University, Stanford, California
}

This article presents system performance studies for the Discovery MI PET/CT system, a new time-of-flight system based on silicon photomultipliers. System performance and clinical imaging were compared between this next-generation system and other commercially available PET/CT and PET/MR systems, as well as between different reconstruction algorithms. Methods: Spatial resolution, sensitivity, noise-equivalent counting rate, scatter fraction, counting rate accuracy, and image quality were characterized with the National Electrical Manufacturers Association NU-2 2012 standards. Energy resolution and coincidence time resolution were measured. Tests were conducted independently on two Discovery MI scanners installed at Stanford University and Uppsala University, and the results were averaged. Back-to-back patient scans were also performed between the Discovery MI, Discovery 690 PET/CT, and SIGNA PET/MR systems. Clinical images were reconstructed using both ordered-subset expectation maximization and Q.Clear (blocksequential regularized expectation maximization with point-spread function modeling) and were examined qualitatively. Results: The averaged full widths at half maximum (FWHMs) of the radial/tangential/ axial spatial resolution reconstructed with filtered backprojection at 1 , 10 , and $20 \mathrm{~cm}$ from the system center were, respectively, 4.10/4.19/ $4.48 \mathrm{~mm}, 5.47 / 4.49 / 6.01 \mathrm{~mm}$, and 7.53/4.90/6.10 mm. The averaged sensitivity was $13.7 \mathrm{cps} / \mathrm{kBq}$ at the center of the field of view. The averaged peak noise-equivalent counting rate was $193.4 \mathrm{kcps}$ at 21.9 $\mathrm{kBq} / \mathrm{mL}$, with a scatter fraction of $40.6 \%$. The averaged contrast recovery coefficients for the image-quality phantom were 53.7, 64.0, 73.1, 82.7, 86.8, and 90.7 for the 10-, 13-, 17-, 22-, 28-, and 37-mmdiameter spheres, respectively. The average photopeak energy resolution was $9.40 \% \mathrm{FWHM}$, and the average coincidence time resolution was 375.4 ps FWHM. Clinical image comparisons between the PET/CT systems demonstrated the high quality of the Discovery MI. Comparisons between the Discovery MI and SIGNA showed a similar spatial resolution and overall imaging performance. Lastly, the results indicated significantly enhanced image quality and contrast-to-noise performance for Q.Clear, compared with ordered-subset expectation maximization. Conclusion: Excellent performance was achieved with the Discovery MI, including 375 ps FWHM coincidence time resolution and sensitivity of $14 \mathrm{cps} / \mathrm{kBq}$. Comparisons between reconstruction algorithms and other multimodal silicon photomultiplier and nonsilicon photomultiplier PET detector system designs indicated that performance can be substantially enhanced with this next-generation system.

Received Jan. 5, 2017; revision accepted Apr. 14, 2017.

For correspondence or reprints contact: Craig S. Levin, 300 Pasteur Dr., Alway Building, Room M001, Stanford, CA 94305.

E-mail: cslevin@stanford.edu

Published online Apr. 27, 2017.

COPYRIGHT (C 2017 by the Society of Nuclear Medicine and Molecular Imaging.
Key Words: PET/CT; NEMA; characterization; instrumentation; image reconstruction

J Nucl Med 2017; 58:1511-1518

DOI: 10.2967/jnumed.117.189514

$\mathbf{P}$

ET is a standard of care for cancer management that has been integrated with CT and MRI. PET/CT is commonly used to differentiate benign from malignant tumors, stage cancer, and plan for radiation therapy (1). Improvements in system performance such as in photon sensitivity, time resolution, and spatial resolution are expected to enhance lesion detection, low-dose patient imaging, and individualized treatment planning and evaluation (2). Many recent commercial PET-based systems have been evaluated using the National Electrical Manufacturers Association (NEMA) NU-2 standards, as previously reported (3-15).

This paper reports on studies performed with the new Discovery MI PET/CT system (GE Healthcare) (16), with the 4-ring PET configuration. The Discovery MI combines a 64 - or 128-slice CT component with a 3- or 4-ring PET component providing a 15- or 20-cm axial field of view (FOV) (16). Each PET ring uses 136 detector blocks, each of which comprises a $4 \times 9$ array of lutetiumyttrium-oxyorthosilicate crystals coupled to a $3 \times 6$ array of silicon photomultipliers (SiPMs) with Anger multiplexing for crystal identification. A closed-loop water cooling system, with real-time local temperature measurement and SiPM gain adjustment capabilities, is used to keep the SiPM arrays at a stable temperature of around $19^{\circ} \mathrm{C}$ in a manner similar to that reported by Levin et al. (17). The crystal elements are $3.95 \mathrm{~mm}$ (transaxial) $\times 5.3 \mathrm{~mm}$ (axial) $\times 25 \mathrm{~mm}$ (length), and each Hamamatsu SiPM array has $2 \times 3$ pixels with an active area of $4 \times 6 \mathrm{~mm}$. A similar detector system design was used in the SIGNA PET/MR system (GE Healthcare) and achieved a coincidence time resolution of less than 400 ps FWHM, making it capable of advanced time-of-flight (TOF) performance (17).

\section{MATERIALS AND METHODS}

NEMA NU-2 2012 testing was performed independently on two Discovery MI systems, one installed at Stanford University and the other at Uppsala University. Before NEMA testing at either site, a well counter calibration scan was performed with ${ }^{18} \mathrm{~F}-\mathrm{FDG}$ in a uniform cylindric phantom. Unless otherwise specified, the testing protocol followed the NEMA NU-2 2012 standards exactly, with results from 
both institutions reported separately and not averaged. Detailed procedures for each test can be found in the NEMA NU-2 2012 standards publication (18).

Because of some interesting technology in this next-generation system, such as use of SiPMs instead of photomultiplier tubes, use of the same detector in both PET/CT and PET/MR systems, a coincidence time resolution of under $400 \mathrm{ps}$, and the new reconstruction algorithms available, we have added to this paper unique comparisons between reconstruction algorithms and other SiPM-based and non-SiPM-based PET/CT and PET/MR detector designs.

\section{Spatial Resolution}

Spatial resolution was measured by creating ${ }^{18} \mathrm{~F}$-FDG point sources using capillary tubes and suspending them at radial offsets of 1,10 , and $20 \mathrm{~cm}$ and axial offsets of 0 and $7.5 \mathrm{~cm}$ from the center of the FOV. Data were collected for $1 \mathrm{~min}$ at each position. The full width at half maximum (FWHM) and full width at tenth maximum of the point sources were quantified at all locations, using the NEMA-specified filtered backprojection algorithm, as well as non-TOF ordered-subset expectation maximization without point-spread function (non-TOF OSEM - PSF) modeling.

\section{Sensitivity}

At both institutions, plastic tubing $(70 \mathrm{~cm}$ in length, $1 \mathrm{~mm}$ in inner diameter) was filled with an averaged calibrated activity of approximately $20 \mathrm{MBq}$ of ${ }^{18} \mathrm{~F}-\mathrm{FDG}$, allowed to decay for $250 \mathrm{~min}$ to reach an activity that generates less than $5 \%$ randoms, and fixed both at the center of the FOV and at a vertical radial offset of $10 \mathrm{~cm}$ using positioning scans and a positioning apparatus. The line source was placed in an aluminum sleeve ensuring complete annihilation of all positrons. Five 1-min scans were taken, adding an additional aluminum sleeve after each measurement.

\section{Counting Rate Statistics}

The patient table was moved to its lowest setting, and the NEMA scatter phantom was propped up to the center of the FOV on stacks of low-density material placed outside the FOV. A line source $(70 \mathrm{~cm}$ in length, $3.2 \mathrm{~mm}$ in inner diameter) was filled with a calibrated activity of roughly $817 \mathrm{MBq}$ of ${ }^{18} \mathrm{~F}-\mathrm{FDG}$ and inserted into the NEMA scatter phantom. Twenty-four frames of data were taken, with the first 17 frames taken as 15-min acquisitions and the last 7 frames taken as 25-min acquisitions followed by 25 -min delays (i.e., at 50-min intervals). NEMA specifications were used to derive the trues, randoms, scatter, and noise-equivalent counting rate (NECR) from the prompts dataset in each frame. Randoms were estimated using singles rates.

\section{Correction Accuracy}

The system's counting rate accuracy, which compares the measured activity with the expected activity and is dependent on the system corrections used, was found from a linear fit of the activity concentrations measured below peak NECR. In addition to attenuation and scatter corrections, randoms and dead-time corrections were performed using singles-based randoms subtraction and pileup correction, respectively. The dimensions of the reconstructed image matrix were $128 \times 128$, with a pixel size of $1.41 \times$ $1.41 \mathrm{~mm}$.

\section{Image Quality}

The background region of the NEMA image-quality phantom and the 10-, 13-, 17-, and 22-mm-diameter spheres were filled with ${ }^{18} \mathrm{~F}$ FDG activity concentrations of, respectively, 4.7 and $18.8 \mathrm{kBq} / \mathrm{mL}$ at Stanford and 5.1 and $20.4 \mathrm{kBq} / \mathrm{mL}$ at Uppsala, yielding a $4: 1$ sphereto-background concentration ratio. The 28- and 37-mm-diameter spheres were filled with nonradioactive water. The scatter line source used to measure NEMA counting rate statistics was filled with roughly $118 \mathrm{MBq}$ of ${ }^{18} \mathrm{~F}-\mathrm{FDG}$ and threaded through the body phantom. For reproducibility, 3 separate acquisitions of the image-quality phantom were taken with decay-adjusted acquisition times of 271,279 , and $282 \mathrm{~s}$, consistent with a 151-mm axial step for each bed position, and reconstructed with the standard GE Healthcare clinical algorithm (TOF OSEM - PSF) and the recently released Q.Clear algorithm (block-sequential regularized expectation maximization + PSF (19)) $(\beta$-value $=50$, yielding noise levels similar to TOF OSEM - PSF $)$.

TABLE 1

Spatial Resolution

\begin{tabular}{|c|c|c|c|c|c|c|c|c|}
\hline \multirow[b]{3}{*}{ Resolution direction } & \multicolumn{4}{|c|}{ Filtered backprojection } & \multicolumn{4}{|c|}{ Non-TOF OSEM - PSF } \\
\hline & \multicolumn{2}{|c|}{ Stanford } & \multicolumn{2}{|c|}{ Uppsala } & \multicolumn{2}{|c|}{ Stanford } & \multicolumn{2}{|c|}{ Uppsala } \\
\hline & FWHM & FWTM & FWHM & FWTM & FWHM & FWTM & FWHM & FWTM \\
\hline \multicolumn{9}{|l|}{$1-\mathrm{cm}$ radial offset } \\
\hline Radial & 4.17 & 9.14 & 4.02 & 8.52 & 3.77 & 7.83 & 3.67 & 7.74 \\
\hline Tangential & 4.40 & 9.17 & 3.97 & 8.19 & 4.00 & 7.95 & 3.74 & 7.93 \\
\hline Axial & 4.57 & 10.38 & 4.39 & 10.12 & 4.00 & 9.80 & 3.93 & 9.71 \\
\hline \multicolumn{9}{|l|}{$10-\mathrm{cm}$ radial offset } \\
\hline Radial & 5.65 & 10.36 & 5.28 & 9.95 & 4.76 & 9.08 & 4.68 & 9.11 \\
\hline Tangential & 4.74 & 9.68 & 4.23 & 8.83 & 4.01 & 8.04 & 3.82 & 7.86 \\
\hline Axial & 6.39 & 12.34 & 5.63 & 11.80 & 5.28 & 8.75 & 4.30 & 9.34 \\
\hline \multicolumn{9}{|l|}{$20-\mathrm{cm}$ radial offset } \\
\hline Radial & 7.52 & 13.88 & 7.54 & 13.38 & 7.36 & 12.99 & 7.44 & 13.27 \\
\hline Tangential & 5.13 & 10.14 & 4.67 & 9.04 & 4.62 & 9.03 & 4.31 & 8.46 \\
\hline Axial & 6.50 & 13.01 & 5.70 & 12.57 & 4.09 & 9.71 & 4.01 & 9.75 \\
\hline
\end{tabular}

FWHM and FWTM (full width at tenth maximum) are in millimeters. 


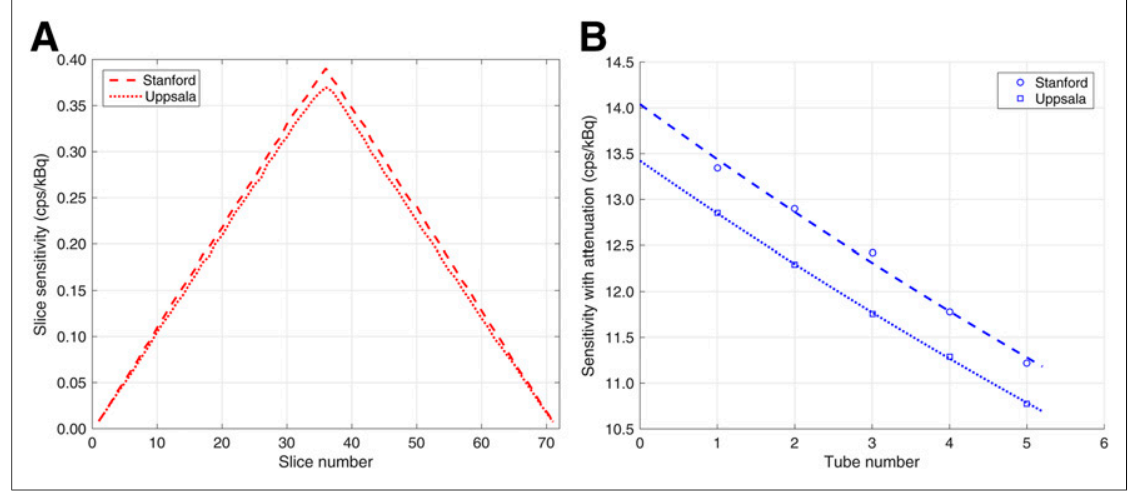

FIGURE 1. NEMA sensitivity measurements. (A) Sensitivity of different axial slices. (B) Sensitivity as function of number of attenuating aluminum sleeves.

The average and SD of the contrast recovery and background variability were quantified over the 3 sets of data replicates. Corrections for randoms, scatter, CT-based attenuation, dead time, and normalization were applied. The dimensions of the reconstructed image matrix were $384 \times$ $384 \times 71$, with a pixel size of $1.042 \times 1.042 \mathrm{~mm}$ and a slice thickness of $2.790 \mathrm{~mm}$.

\section{Energy and Timing Resolutions}

A line source $70 \mathrm{~cm}$ in length was filled with $12 \mathrm{MBq}$ of ${ }^{18} \mathrm{~F}-\mathrm{FDG}$ solution and suspended at the center of the FOV in the axial direction inside the smallest aluminum sleeve used in the NEMA sensitivity measurement. To acquire the timing and energy spectra, 400 million coincident counts were taken. Measurement of the timing resolution FWHM was based on a 3-point fit of the peak for each crystal pair's timing spectra after randoms were removed. For the energy resolution, the energy spectra were smoothed with a boxcar filter, and the percentage FWHM per channel was measured. The system energy and timing resolutions were quantified by averaging the values from all the detector crystals and channels in the system.

\section{Comparison with Discovery 690}

To study the potential benefits of SiPM technology, we compared the performance of the Discovery MI with that of a photomultipliertube-based PET/CT system, the Discovery 690 (GE Healthcare), at Stanford University. A patient with a history of melanoma who required restaging and metastatic evaluation was injected with $298 \mathrm{MBq}$ of ${ }^{18} \mathrm{~F}-\mathrm{FDG}$, and a 33-min total-body scan was taken $96 \mathrm{~min}$ afterward on the Discovery 690. At roughly $130 \mathrm{~min}$ after injection, another 33-min total-body scan was taken, this time on the Discovery MI. Singles-estimate randoms correction, CT-based scatter and attenuation correction, and dead-time correction were applied. Image reconstruction for both systems used TOF OSEM - PSF with 3 iterations, 16 subsets, and a 5-mm postprocessing filter, as well as Q.Clear with a $\beta$-value of 350 , which was chosen to match the noise level in TOF OSEM - PSF reconstructions.

\section{Comparison with SIGNA}

Because the same basic SiPM-based detector design is incorporated in both the Discovery MI system and the SIGNA PET/ MR system (GE Healthcare), we are in the unique position of being able to compare the PET performance in CT versus MR environments. A neuroendocrine tumor patient was scanned with ${ }^{68}$ Ga-DOTATOC on the Discovery MI and SIGNA systems over 2 consecutive days at Uppsala University. For the Discovery MI, the scan began $72 \mathrm{~min}$ after injection of $181 \mathrm{MBq}$ of ${ }^{68} \mathrm{Ga}$-DOTATOC and proceeded at a rate of $2 \mathrm{~min}$ per bed position. For the SIGNA, the scan began $69 \mathrm{~min}$ after injection of $148 \mathrm{MBq}$ of ${ }^{68} \mathrm{Ga}$-DOTATOC and proceeded at a rate of $3 \mathrm{~min}$ per bed position. Image reconstruction for both systems used TOF OSEM with PSF (TOF OSEM + PSF) with 3 iterations, 16 subsets, and a 5-mm postprocessing filter. In addition, the Discovery MI images were reconstructed using Q.Clear with a $\beta$-value of 350 . Attenuation correction was performed using a standard 2-point Dixon MR sequence.

\section{RESULTS}

\section{Spatial Resolution}

Table 1 shows the spatial resolution results for both filtered backprojection and non-TOF OSEM - PSF.

\section{Sensitivity}

At the center of the FOV, sensitivity was $14.0 \mathrm{cps} / \mathrm{kBq}$ at Stanford and $13.4 \mathrm{cps} / \mathrm{kBq}$ at Uppsala. At a radial offset of $10 \mathrm{~cm}$, sensitivity was $13.8 \mathrm{cps} / \mathrm{kBq}$ at Stanford and $13.1 \mathrm{cps} / \mathrm{kBq}$ at Uppsala. Figure $1 \mathrm{~A}$ shows the slice sensitivity profile at the center of the FOV, and Figure 1B shows the extrapolation process used to remove attenuation from the aluminum tubes in order to obtain attenuation-free sensitivity numbers.
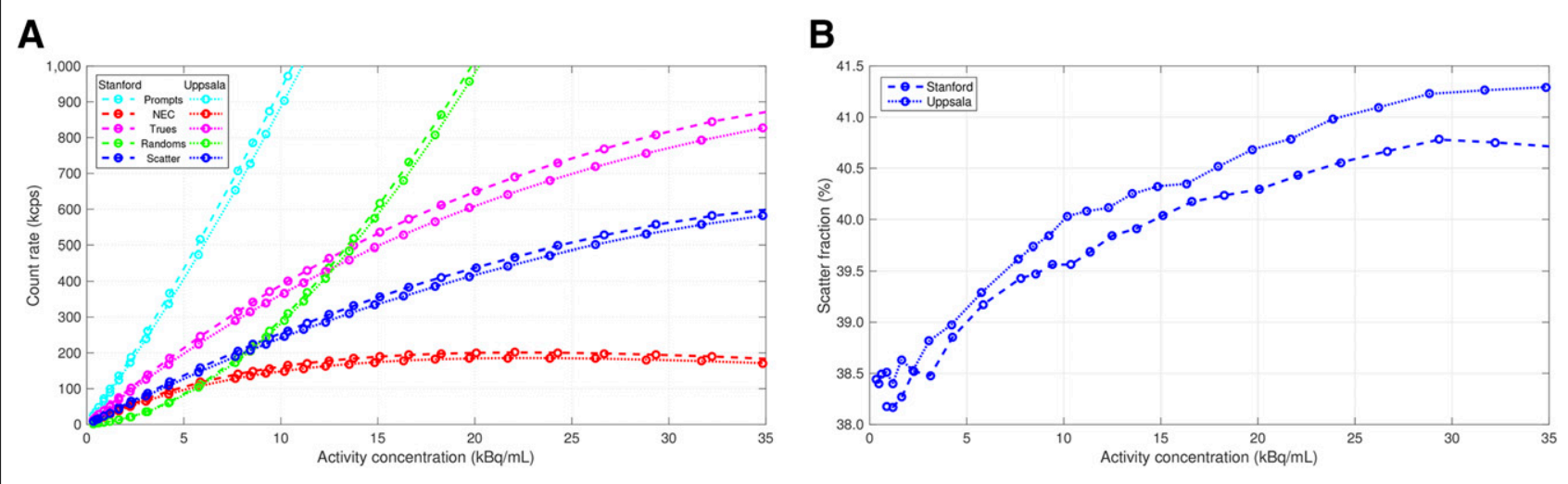

FIGURE 2. NEMA counting rate measurements. (A) Counting rate vs. activity. (B) Scatter fraction vs. activity. NEC = noise-equivalent counts. 
TABLE 2

Counting Rate Measurements

\begin{tabular}{lrr}
\hline \multicolumn{1}{c}{ Type of measurement } & Stanford & Uppsala \\
\hline Peak NECR $(\mathrm{kcps})$ & 201.1 & 185.7 \\
\hline Activity at peak NECR $(\mathrm{kBq} / \mathrm{mL})$ & 22.1 & 21.7 \\
\hline Peak true counting rate $(\mathrm{kcps})$ & 875.9 & 827.0 \\
\hline $\begin{array}{l}\text { Activity at peak true counting } \\
\text { rate }(\mathrm{kBq} / \mathrm{mL})\end{array}$ & 35.4 & 34.8 \\
\hline Scatter fraction at peak NECR $(\%)$ & 40.4 & 40.8 \\
\hline
\end{tabular}

\section{Counting Rate Statistics}

Figure $2 \mathrm{~A}$ shows the trues, randoms, scatters, NECR, and totalevent rate as a function of activity, and Figure $2 \mathrm{~B}$ shows the system scatter fraction as a function of activity. Table 2 summarizes important counting rate metrics measured at both Stanford University and Uppsala University.

\section{Correction Accuracy}

The average counting rate error at both institutions, defined by the percentage deviation from the expected activity concentration, is shown in Figure 3 as a function of the activity in the line source. The error bars indicate the maximum positive and negative slice deviations from the average error at each activity level. The maximum deviation from expected activity at Uppsala was $3.86 \%$ at an activity of $1.19 \mathrm{kBq} / \mathrm{mL}$, whereas at Stanford it was $2.43 \%$ at an activity of $35.44 \mathrm{kBq} / \mathrm{mL}$.

\section{Image Quality}

The image contrast recovery and background variability of the image-quality phantom are shown in Table 3 and Figure 4. The average lung error for TOF OSEM - PSF was $4.4 \% \pm 0.1 \%$ at Stanford and $5.3 \% \pm 0.0 \%$ at Uppsala, whereas for Q.Clear it was $2.5 \% \pm 0.1 \%$ at Stanford and $3.3 \% \pm 0.1 \%$ at Uppsala. The reconstructed transverse and coronal images from the image-quality phantom are shown in Figure 5 and reveal the better noise and contrast recovery performance of Q.Clear.

\section{Energy and Timing Resolutions}

The average system photopeak energy resolution was $9.44 \% \pm$ $0.07 \%$ FWHM at Stanford and $9.35 \% \pm 0.05 \%$ FWHM at Uppsala. The average system coincidence time resolution was $374.1 \pm 2.6 \mathrm{ps}$ FWHM at Stanford and 376.7 \pm 2.7 ps FWHM at Uppsala.

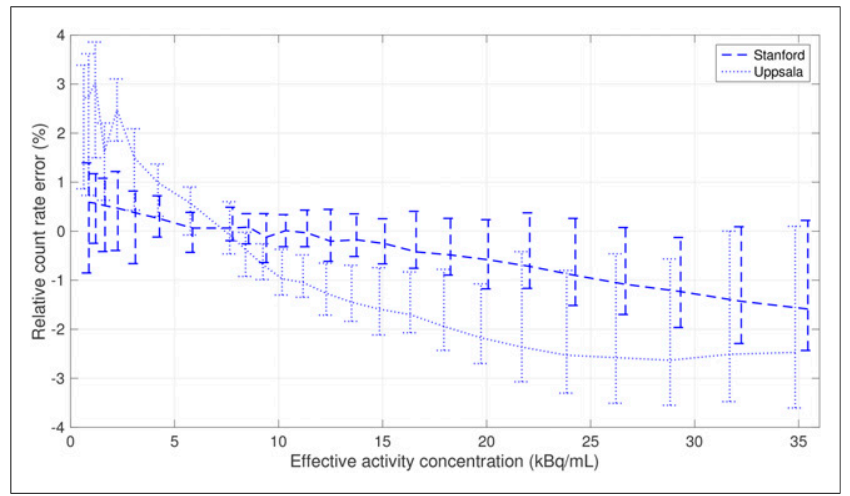

FIGURE 3. Counting rate error measured as percentage deviation from expected activity concentration. Error bars show range of deviations from expected activity level.
TABLE 3

Contrast Recovery and Background Variability

\begin{tabular}{|c|c|c|c|c|}
\hline \multirow{2}{*}{$\begin{array}{l}\text { Sphere diameter } \\
\qquad(\mathrm{mm})\end{array}$} & \multicolumn{2}{|c|}{ TOF OSEM - PSF } & \multicolumn{2}{|c|}{ Q.Clear } \\
\hline & CR (\%) & BV (\%) & CR (\%) & BV (\%) \\
\hline \multicolumn{5}{|l|}{ Stanford } \\
\hline 10 & $51.7 \pm 2.3$ & $10.2 \pm 1.3$ & $67.2 \pm 5.1$ & $7.9 \pm 0.7$ \\
\hline 13 & $61.5 \pm 6.2$ & $7.8 \pm 0.7$ & $72.5 \pm 4.4$ & $6.2 \pm 0.4$ \\
\hline 17 & $66.2 \pm 2.0$ & $6.0 \pm 0.2$ & $74.4 \pm 0.8$ & $4.8 \pm 0.1$ \\
\hline 22 & $81.3 \pm 2.1$ & $4.8 \pm 0.2$ & $87.7 \pm 0.8$ & $3.8 \pm 0.4$ \\
\hline 28 & $86.6 \pm 1.8$ & $3.8 \pm 0.1$ & $91.1 \pm 0.6$ & $2.9 \pm 0.3$ \\
\hline 37 & $90.0 \pm 1.4$ & $3.0 \pm 0.2$ & $94.0 \pm 0.5$ & $2.4 \pm 0.3$ \\
\hline \multicolumn{5}{|l|}{ Uppsala } \\
\hline 10 & $55.6 \pm 1.5$ & $10.6 \pm 0.1$ & $72.9 \pm 2.7$ & $8.5 \pm 1.0$ \\
\hline 13 & $66.5 \pm 2.6$ & $8.1 \pm 0.3$ & $80.2 \pm 2.9$ & $6.3 \pm 0.4$ \\
\hline 17 & $80.1 \pm 3.8$ & $5.8 \pm 0.3$ & $87.1 \pm 2.6$ & $4.7 \pm 0.1$ \\
\hline 22 & $84.1 \pm 2.6$ & $4.3 \pm 0.5$ & $90.8 \pm 1.3$ & $3.7 \pm 0.3$ \\
\hline 28 & $86.9 \pm 1.9$ & $3.7 \pm 0.4$ & $91.8 \pm 0.8$ & $3.0 \pm 0.4$ \\
\hline 37 & $91.4 \pm 0.4$ & $2.9 \pm 0.3$ & $94.5 \pm 0.3$ & $2.5 \pm 0.3$ \\
\hline
\end{tabular}

$\mathrm{CR}=$ contrast recovery; $\mathrm{BV}=$ background variability .

\section{Comparison with Discovery 690}

Figure 6 shows maximum-intensity-projection coronal PET slices, axial PET slices, and axial PET/CT slices for the Discovery MI and the Discovery 690 using TOF OSEM - PSF and Q.Clear. Because the two systems have different axial slice thicknesses, the displayed images were carefully chosen to ensure the best alignment possible.

\section{Comparison with SIGNA}

Figure 7 shows coronal-slice and maximum-intensity-projection images for the Discovery MI and the SIGNA using TOF OSEM + PSF and Q.Clear. The displayed images were carefully chosen to ensure the closest possible alignment and intensity scales between the two systems.

\section{DISCUSSION}

\section{Intersystem Variance}

Differences in the manufacturing process may explain general trends in variance between the Discovery MI at Stanford and the Discovery MI at Uppsala, such as the better counting rate performance and worse contrast recovery performance of the former. However, differences in the phantom-filling process might also have caused some of the variance in results. Imperfect filling of the 17-mm sphere at Stanford could explain its lower contrast recovery numbers, and differences in the axial confinement of the capillary tube could explain differences in axial spatial resolution. The lower sensitivity measured at Uppsala might also have been caused by variations in activity calibration.

\section{Performance Measurement Observations}

Because of the high photon sensitivity of the Discovery MI and the low clinical dosage recommended, NECR testing did not reach the system's peak trues counting rate and activity. 


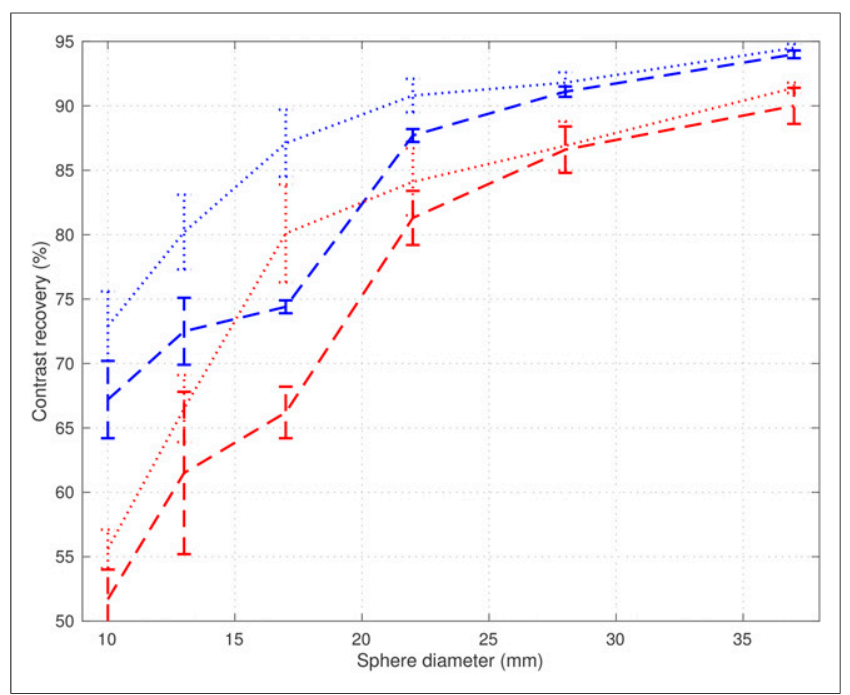

FIGURE 4. Contrast recovery and background variability measured with TOF OSEM - PSF (red lines) and Q.Clear (blue lines) at Stanford (dashed lines) and Uppsala (dotted lines).

The amount of activity required would be so high as to require sweeping a large nonclinically relevant portion of the NECR curve. Similarly, the measured maximum relative counting rate error at both institutions occurred at activity levels that are not clinically relevant, whereas the relative counting rate error was less than $3 \%$ at both institutions for clinically relevant activity levels. A low relative counting rate error is clinically important for accurate lesion quantization, posttreatment follow-up scans, and longitudinal studies. The background activity concentration used in the image-quality phantom at Stanford, 0.6 $\mathrm{kBq} / \mathrm{mL}$, was lower than the NEMA specifications. This factor leads to a more conservative contrast recovery measurement due to a lower number of acquired counts and could explain why the contrast recovery measured for the smaller spheres was $8 \%$ lower for Stanford's system than for Uppsala's. A reduction in the system's axial FOV from 20 to $15 \mathrm{~cm}$ (3-ring configuration) would result in a reduced sensitivity of $7.5 \mathrm{kcps} / \mathrm{MBq}$ and peak NECR of $100 \mathrm{kcps}$ at $20.6 \mathrm{kBq} / \mathrm{mL}$ (16). The measured energy and timing resolutions can be improved using lower activity levels.

\section{Clinical System Comparisons}

Figure 6 shows that regardless of reconstruction method, several lesions were visible in the Discovery MI images but not in the Discovery 690 images. The likely cause is the Discovery MI's improved sensitivity, NECR, timing resolution, and contrast recovery for small lesions, when compared with the Discovery 690, as shown in Table 4. However, direct comparisons cannot be made because of the differences in tracer uptake time and patient alignment between the two acquisitions. Comparing Q.Clear with the more traditional TOF OSEM - PSF, the intensity of lesion uptake is clearly higher for the former, with better-defined lesion boundaries. This improvement can help in surgery and radiotherapy planning or in tumor segmentation for quantitative studies on treatment effectiveness.

Likewise, direct comparisons cannot be made between the Discovery MI and the SIGNA because the patient was scanned on 2 consecutive days with slightly different amounts of activity and scanning times per bed position. However, it is clear from Figure 7 that, unsurprisingly, lesion identification capability and spatial resolution are similar between the Discovery MI and the SIGNA since they use similar block detector architectures (17). In addition, lesion visualization near the center of the body does not significantly differ between the CT-based and MR-based attenuation correction of the two systems. Comparison of the TOF OSEM + PSF coronal slices shows better noise performance for the Discovery MI than for the SIGNA, making lesion identification easier for the former. The use of Q.Clear reconstruction instead of OSEM-based reconstruction dramatically suppresses background noise while having minimal effects on lesion intensity.

\section{Comparisons with Other Multimodal Systems}

Table 4 compares published NEMA NU-2 measurements for some of the newest commercial multimodal PET scanners. The Discovery MI metrics in this table are averages of the NEMA NU2 results from Stanford and Uppsala. The Discovery MI, SIGNA, Biograph mCT Flow (Siemens Healthcare), and Vereos Digital (Philips Healthcare) were tested using the NEMA NU-2 2012 standards, and the remaining systems were tested with the NEMA NU-2 2007 standards.

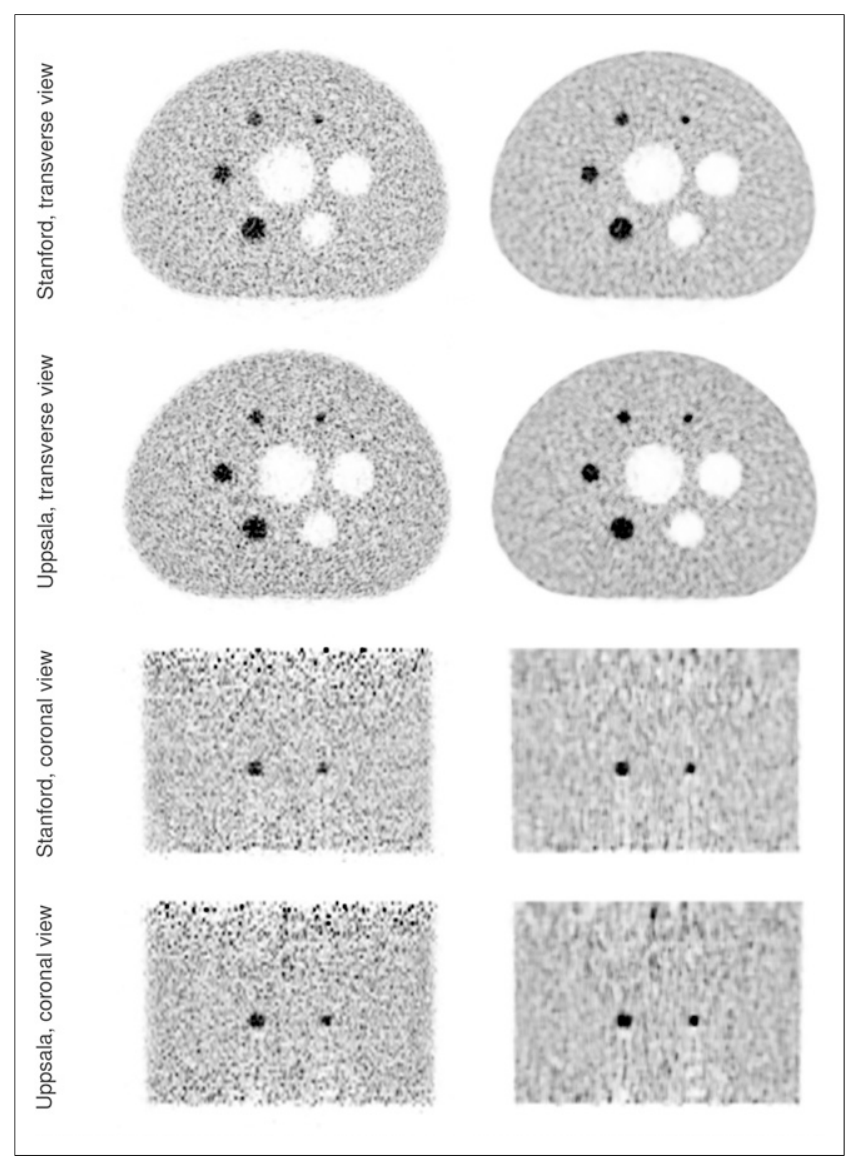

FIGURE 5. Image-quality phantom images reconstructed with TOF OSEM - PSF (left) and Q.Clear (right). Top two rows show transverse slices through center of all spheres. Bottom two rows show coronal slices through $10 / 13$-mm spheres. 


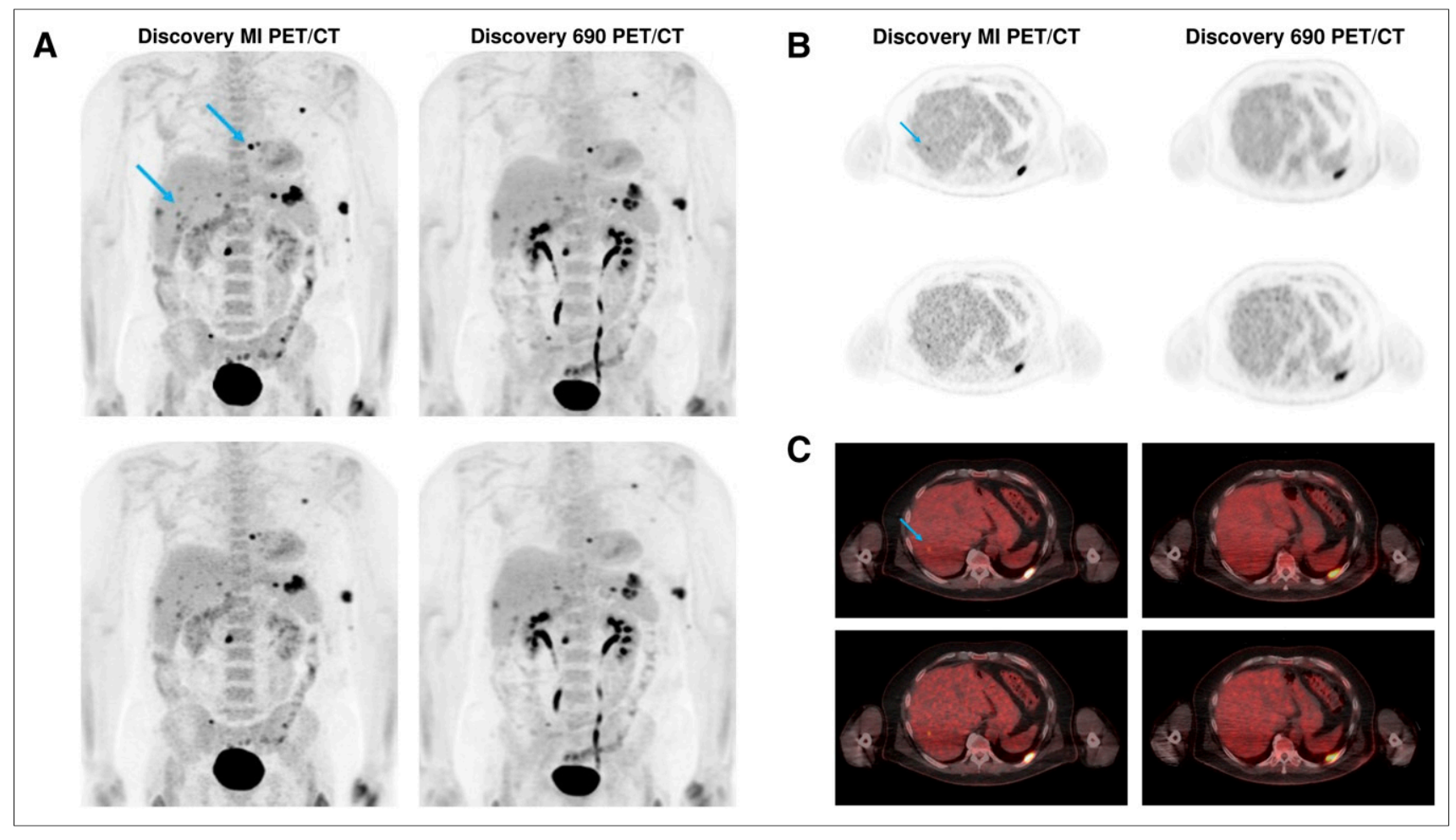

FIGURE 6. Melanoma patient scanned on both Discovery 690 and Discovery MI, with images reconstructed using Q.Clear (top row in each panel) and TOF OSEM - PSF (bottom row in each panel). Arrows indicate small metastases visible on Discovery MI but not on Discovery 690. (A) Maximum-intensity projection coronal PET images. (B) Transaxial PET images. (C) Transaxial PET/CT images.

Comparisons of NEMA NU-2 2007 and NEMA NU-2 2012 must take into account slight differences between the two sets of standards. For spatial resolution tests, the 2007 standards do not report radial offsets of $20 \mathrm{~cm}$ or separate radial and tangential resolutions at the center of the FOV, and the 2007 standards average the resolutions of 2 sources at $10-\mathrm{cm}$ radial offsets in

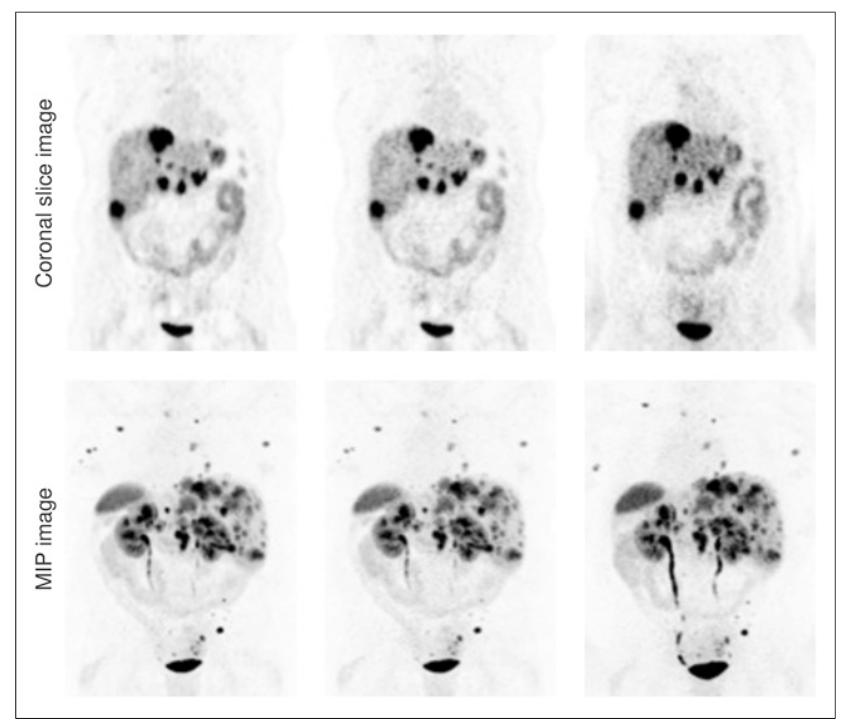

FIGURE 7. Reconstructed coronal and maximum-intensity-projection (MIP) images from Discovery MI (first and second columns) and SIGNA (third column). Second and third columns were reconstructed with TOF OSEM + PSF, and first column was reconstructed with Q.Clear. orthogonal directions. For NECR tests, the 2007 standards do not allow shimming of the phantom, leading to larger table scatter contribution for systems with a larger range of table height adjustment. The counting rate accuracy measured by the 2007 standards uses extrapolation over the lowest 3 measurement points instead of over all points below peak NECR, and the 2007 standards also do not require corrections to be performed on reconstructed images - a requirement that can be challenging for scatter models that vary with counting rate. Most significantly, image-quality measurements in the 2007 standards use acquisitions that are twice as long as in the 2012 standards, leading to artificially favorable contrast recovery and background variability for the 2007 standards.

The spatial resolution testing showed that, taken as a whole over all 3 resolution directions and the different distances from the center of the FOV, the Discovery MI performs comparably to the other systems in Table 4.

The sensitivity of the Discovery MI is the highest of all the PET/CT systems although still lower than that of the PET/MR systems, with longer PET axial FOVs and smaller transaxial FOVs. A higher sensitivity allows for a better signal-to-noise ratio for a given acquisition time or the same signal-to-noise ratio for shortened acquisitions or reduced patient doses. The counting rate performance of the Discovery MI reinforces this observation.

Compared with the Discovery 690, the Discovery MI has a peak NECR that is $39 \%$ higher and a peak noise-equivalent-count activity concentration that is $24 \%$ lower. The higher peak NECR allows for the same signal-to-noise ratio in a $39 \%$ shorter acquisition, and even more so when accounting for the improved timing resolution and associated TOF signal-to-noise gain of the 
TABLE 4

NEMA NU-2 Measurements for Other Commercial PET Scanners

\begin{tabular}{|c|c|c|c|c|c|c|c|}
\hline \multirow[b]{2}{*}{ Parameter } & \multicolumn{3}{|c|}{ GE Healthcare } & \multicolumn{2}{|c|}{ Siemens Healthcare } & \multicolumn{2}{|c|}{ Philips Healthcare } \\
\hline & $\begin{array}{c}\text { Discovery MI } \\
\text { PET/CT } \\
\text { (this work) }\end{array}$ & $\begin{array}{c}\text { SIGNA } \\
\text { PET/MR (12) }\end{array}$ & $\begin{array}{c}\text { Discovery } \\
690 / 710 \\
\text { PET/CT (4) }\end{array}$ & $\begin{array}{l}\text { Biograph } \\
\text { mCT Flow } \\
\text { PET/CT (7) }\end{array}$ & $\begin{array}{c}\text { Biograph } \\
\text { mMR } \\
\text { PET/MR (14) }\end{array}$ & $\begin{array}{c}\text { Vereos } \\
\text { Digital } \\
\text { PET/CT (20) }\end{array}$ & $\begin{array}{l}\text { Ingenuity } \\
\text { TF } 128 \\
\text { PET/CT (8) }\end{array}$ \\
\hline Axial FOV (cm) & 20 & 25 & 15.7 & 22.1 & 25.8 & 16.4 & 18 \\
\hline Transverse FOV (cm) & 70 & 60 & 70 & 70 & 59.4 & 67.6 & 67.6 \\
\hline Detector ring diameter $(\mathrm{cm})$ & 74.4 & 62.4 & 81.0 & 84.2 & 65.6 & 76.4 & 90 \\
\hline Crystal thickness (mm) & 25 & 25 & 25 & 20 & 20 & 19 & 22 \\
\hline \multicolumn{8}{|l|}{ Spatial resolution $\mathrm{FWHM}^{*}$} \\
\hline Radial, $1 \mathrm{~cm}$ & 4.10 & 4.46 & $4.70^{\dagger}$ & $4.33^{\dagger}$ & $4.3^{\dagger}$ & $4.01^{\dagger}$ & $4.84^{\dagger}$ \\
\hline Tangential, $1 \mathrm{~cm}$ & 4.19 & 4.08 & $4.70^{\dagger}$ & $4.33^{\dagger}$ & $4.3^{\dagger}$ & $4.01^{\dagger}$ & $4.84^{\dagger}$ \\
\hline Axial, $1 \mathrm{~cm}$ & 4.48 & 5.35 & 4.74 & 4.25 & 4.3 & 4.14 & 4.73 \\
\hline Radial, $10 \mathrm{~cm}$ & 5.47 & 5.81 & 5.34 & 5.16 & 5.2 & NA & 5.25 \\
\hline Tangential, $10 \mathrm{~cm}$ & 4.49 & 4.44 & 4.79 & 4.72 & 4.8 & NA & 5.01 \\
\hline Axial, $10 \mathrm{~cm}$ & 6.01 & 6.75 & 5.55 & 5.85 & 6.6 & NA & 5.23 \\
\hline Radial, $20 \mathrm{~cm}$ & 7.53 & 8.42 & NA & 5.55 & NA & $5.82^{\dagger}$ & NA \\
\hline Tangential, $20 \mathrm{~cm}$ & 4.90 & 5.27 & NA & 6.48 & NA & $5.82^{\dagger}$ & NA \\
\hline Axial, $20 \mathrm{~cm}$ & 6.10 & 7.30 & NA & 7.80 & NA & 6.17 & NA \\
\hline Center of FOV (cps/kBq) & $13.7\left(7.5^{\ddagger}\right)$ & 22.9 & 7.4 & $9.6\left(5.4^{\S}\right)$ & 15.0 & 5.7 & 7.39 \\
\hline \multicolumn{8}{|l|}{ Counting rate statistics } \\
\hline Peak NECR (kcps) & 193.4 & 214.8 & 139.1 & 185 & 184 & 171 & 124.1 \\
\hline Peak NEC activity (kBq/mL) & 21.9 & 17.6 & 29.0 & 29.0 & 23.1 & 50 & 20.3 \\
\hline Peak NEC scatter fraction (\%) & 40.6 & 42.5 & 37 & 33.5 & 37.9 & 30 & 36.7 \\
\hline Maximum absolute error (\%) & 3.14 & 3.5 & 2.09 & 3.7 & 5.5 & NA & NA \\
\hline \multicolumn{8}{|l|}{ Contrast recovery in spheres } \\
\hline $10 \mathrm{~mm}$ & 53.7 & 36.5 & 44 & 28.5 & 32.5 & 62 & 17 \\
\hline $13 \mathrm{~mm}$ & 64.0 & 50.6 & 56 & 42.3 & 50.0 & NA & 46 \\
\hline $17 \mathrm{~mm}$ & 73.1 & 60.0 & 65 & 58.4 & 62.9 & NA & 58 \\
\hline $22 \mathrm{~mm}$ & 82.7 & 68.6 & 75 & 71.7 & 70.8 & 88 & 63 \\
\hline $28 \mathrm{~mm}$ & 86.8 & 80.7 & 87 & 70.1 & 65.1 & 86 & 68 \\
\hline $37 \mathrm{~mm}$ & 90.7 & 88.6 & 89 & 78.3 & 72.3 & 89 & 68 \\
\hline Timing resolution (ps) & 375.4 & 390 & 544.3 & $555^{\|}$ & 2,930 & 322 & 502 \\
\hline Energy resolution (\%) & 9.40 & 10.5 & 12.4 & NA & 14.5 & 11.0 & 11.1 \\
\hline
\end{tabular}

${ }^{*}$ Filtered backprojection.

${ }^{\dagger}$ Radial and tangential values are averaged.

FWith 15-cm axial FOV (16).

${ }^{5}$ With 16.2-cm axial FOV (11).

"Value is unclear in reference.

$\mathrm{NA}=$ not applicable; NEC = noise-equivalent count.

For each column, numeric precisions are exactly as presented in that reference. The Discovery 690 and 710 have same PET subsystem, so the NEMA results apply to both. The Vereos Digital is not yet commercially available.

Discovery MI. The lower peak noise-equivalent-count activity concentration points to the viability of the Discovery MI as a lowdose imaging system.

The contrast recovery for small spheres is better for the Discovery MI than for any of the other commercially available systems in Table 4. Compared with the Discovery 690, which has the next best contrast recovery, that of the Discovery MI is $22 \%$ better for the 10 -mm sphere, $14 \%$ better for the 13 -mm sphere, $12 \%$ better for the 17-mm sphere, and $11 \%$ better for the $22-\mathrm{mm}$ sphere. This better contrast recovery should lead to an improvement in the system's ability to detect, visualize, and quantify smaller lesions.

\section{CONCLUSION}

NEMA NU-2 2012 testing of the SiPM-based Discovery MI PET/CT systems at Stanford University and Uppsala University points to improved diagnostic sensitivity for small lesions and a 
wide range of promising applications, from low-dose oncology studies to high-dose studies with short-lived isotopes. In addition, comparisons with other PET/CT and PET/MR systems demonstrate the substantial performance improvements possible with the new generation of SiPM-based TOF PET/CT systems.

\section{DISCLOSURE}

Craig Levin is participating in a sponsored research agreement with GE Healthcare on an unrelated topic. No other potential conflict of interest relevant to this article was reported.

\section{ACKNOWLEDGMENTS}

For valuable contributions in the data collection process for this publication, we thank Adam Brown, Jakub Siennicki, and Tarik Cengiz from GE Healthcare; Monica Ranger and Vinh Nguyen from Stanford Healthcare; Dr. Fred Chin and the Radiochemistry Group at Stanford; and Lars Lindsjö and Prof. Anders Sundin from Uppsala University Hospital. We also thank Dr. Andrei Iagaru, for coordinating the clinical studies and the world's first installation of the Discovery MI system at Stanford University.

\section{REFERENCES}

1. Boellaard R, Delgado-Bolton R, Oyen WJG, et al. FDG PET/CT: EANM procedures guidelines for tumour imaging: version 2.0. Eur J Nucl Med Mol Imaging. 2015;42:328-354.

2. Ell PJ. The contribution of PET/CT to improved patient management. $\mathrm{Br} \mathrm{J}$ Radiol. 2006;79:32-36.

3. De Ponti E, Morzenti S, Guerra L, et al. Performance measurements for the PET/ CT Discovery-600 using NEMA NU 2-2007 standards. Med Phys. 2011;38:968974.

4. Bettinardi V, Presotto L, Rapisarda E, Picchio M, Gianolli L, Gilardi MC. Physical performance of the new hybrid PET/CT Discovery-690. Med Phys. 2011;38:5394 5411.

5. Martí-Climent JM, Prieto E, Dominguez-Prado I, et al. Contribution of time of flight and point spread function modeling to the performance characteristics of the PET/CT Biograph mCT scanner [in Spanish]. Rev Esp Med Nucl Imagen Mol. 2013;32:13-21
6. Jakoby BW, Bercier Y, Conti M, Casey ME, Bendriem B, Townsend DW. Physical and clinical performance of the mCT time-of-flight PET/CT scanner. Phys Med Biol. 2011;56:2375-2389.

7. Rausch I, Cal-González J, Dapra D, et al. Performance evaluation of the Biograph mCT Flow PET/CT system according to the NEMA NU2-2012 standard. EJNMMI Phys. 2015;2:26.

8. Kolthammer JA, Su KH, Grover A, Narayanan M, Jordan DW, Muzic RF. Performance evaluation of the Ingenuity TF PET/CT scanner with a focus on high count-rate conditions. Phys Med Biol. 2014;59:3843-3859.

9. Surti S, Kuhn A, Werner ME, Perkins AE, Kolthammer J, Karp JS. Performance of Philips Gemini TF PET/CT scanner with special considerations for its timeof-flight imaging capabilities. J Nucl Med. 2007;48:471-480.

10. Grogg KS, Toole T, Ouyang J, et al. National Electrical Manufacturers Association and clinical evaluation of a novel brain PET/CT scanner. J Nucl Med. 2016;57:646-652.

11. Akamatsu G, Uba K, Taniguchi T, et al. Impact of time-of-flight PET/CT with a large axial field of view for reducing whole-body acquisition time. $\mathrm{J} \mathrm{Nucl} \mathrm{Med}$ Technol. 2014;42:101-104.

12. Grant AM, Deller TW, Khalighi MM, Maramraji SH, Delso G, Levin CS. NEMA NU 2-2012 performance studies for the SiPM-based ToF-PET component of the GE SIGNA PET/MR system. Med Phys. 2016;43:2334-2343.

13. Karlberg AM, Sæther O, Eikenes L, Goa PE. Quantitative comparison of PET performance: Siemens Biograph mCT and mMR. EJNMMI Phys. 2016;3:5.

14. Delso G, Fürst S, Jakoby B, et al. Performance measurements of the Siemens mMR integrated whole-body PET/MR scanner. J Nucl Med. 2011;52:19141922.

15. Teoh EJ, McGowan DR, Macpherson RE, Bradley KM, Gleeson FV. Phantom and clinical evaluation of the Bayesian penalized likelihood reconstruction algorithm Q.Clear on an LYSO PET/CT system. J Nucl Med. 2015;56:14471452 .

16. Levin CS, Peterson W, Ross S, Stearns C, Uribe J. PET performance as a function of axial field of view for a new silicon photomultiplier-based whole body TOF PET/CT system [abstract]. J Nucl Med. 2016;57(suppl 2):200.

17. Levin CS, Maramraji SH, Khalighi MM, Deller TW, Delso G, Jansen F. Design features and mutual compatibility studies of the time-of-flight PET capable GE SIGNA PET/MR system. IEEE Trans Med Imaging. 2016;35:1907-1914.

18. NEMA NU-2 2012: Performance Measurement of Positron Emission Tomographs. Rosslyn, VA: National Electrical Manufacturers Association; 2013.

19. Lantos J, Iagaru A, Levin CS. Scanner dependent noise properties of the Q.Clear PET image reconstruction tool. IEEE Xplore Digital Library website. http://ieeexplore.ieee.org/document/7582176/. Published 2015. Accessed May $16,2017$.

20. Zhang J, Miller M, Knopp MV. SU-F-I-55: Performance evaluation of digital PET/CT-medical physics basis for the clinical applications. Med Phys. 2016;43:3399 